\title{
The symbolic distance effect in monkeys (Cebus apella)
}

\author{
M. R. D'AMATO and M. COLOMBO \\ Rutgers-The State University, New Brunswick, New Jersey
}

\begin{abstract}
In making comparative judgments about pairs of stimuli that are linearly ordered, human subjects usually respond faster the greater the separation between the items of a test pair-the symbolic distance effect. A similar result has been obtained for associatively related items, such as the alphabet. We report evidence for a distance effect in monkeys tested with pairs of items drawn from a five-item series with which they had considerable previous experience in a serial learning setting. This finding provides independent evidence that in learning a serial list of items, monkeys acquire knowledge about the ordinal positions of the items. Analysis of the positive results obtained in Experiment 2 and of the failure to find a distance effect in Experiment 1 suggested that in learning a serial list, monkeys construct both an associative chain representation of the series and a spatial representation, with the latter supplying the spatial markers that convey positional information. This dual coding of sequential events, which may be rather general among mammals, probably supports a variety of cognitive competencies.
\end{abstract}

The capacity of animals to represent events and the nature of such assumed internal representations are important, if contentious, issues for comparative cognition (see Roitblat, 1982, and the accompanying commentaries). During the last few years, we have focused on one facet of this complex problem area-the monkey's representation of serially ordered events.

Following the lead of earlier work with pigeons (e.g., Straub \& Terrace, 1981), we trained cebus monkeys to respond to five visual stimuli in a specific order, symbolized as ABCDE. The spatial configuration of the five stimuli changed from one trial to another and all stimuli remained present until an error was committed or the sequence was successfully completed. Thus, no information regarding the correct serial order was available in the stimulus array. The monkeys nevertheless learned the task within a reasonable number of training sessions, and to a high level of accuracy (D'Amato \& Colombo, 1988).

Several features of the results suggested that the subjects had developed an internal representation of the series that could be accessed to guide their instrumental behavior. During the terminal training trials, the monkeys executed the sequence very rapidly, as though their only task was to map the items of the stimulus array onto an internal analogue of the sequence. A subsequent pairwise test also proved revealing. Of the 10 possible pairs of items that can be generated from a five-item series, one pair was presented on each test trial. The subject's task was to respond to the two items in the order appropriate for the full sequence. Given Test Pair BE, for example,

This research was supported by National Science Foundation Grant 8417383. Correspondence concerning this article may be addressed to M. R. D'Amato, Department of Psychology, Rutgers-The State University, New Brunswick, NJ 08903. the subjects had to respond first to B and then to E. In contrast to pigeons administered a similar test after training on a four-item (Straub \& Terrace, 1981) or a fiveitem (Terrace, 1987, Groups I, III, V) series, the monkeys were able to respond appropriately to the interior pairs of items (BC, BD, and $\mathrm{CD}$ ). Competence on interior pairs is to be expected if the monkeys had indeed formed a functional representation of the series.

There was, moreover, a strong monotonic relationship between response latency to the first item of a pair and the item's position in the series, with the shortest firstitem response latency being observed for pairs that included Item A. It seemed that when confronted with a test pair, the monkeys accessed their representation of the sequence at Item $\mathrm{A}$, progressing along the sequence until they found a match between a represented and a displayed item. Supporting such an interpretation, response latency to the second item of a test pair increased monotonically as the number of missing items separating the first and second items increased. Thus, for example, response latency to Item D was longer for Test Pair BD than for Pair CD.

We pointed out that all of the above results could be accommodated by assuming that the internal representation of the five-item series acquired by the monkeys consisted of a simple associative chain that linked together the separate representations of the series' items. However, a major limitation of such chaining accounts is that they do not allow for knowledge of ordinal position. As observed by Slamecka (1985) in the context of human serial learning, such interpretations eliminate the need for constructs having to do with acquisition of order information, for it is "associations that are literally acquired rather than some abstract knowledge of what follows what" (p. 417). 
Phrasing this distinction in somewhat different terms, the only knowledge regarding sequential position granted to the subject by the associative chain account is that which can be acquired from serial access to the items of a sequence; there is no ordinal information attached to the items themselves. Referring to the alphabet, for example, by this view a subject knows that the letter $\mathrm{H}$ comes before $\mathrm{R}$ only by accessing the alphabet at $\mathrm{A}$ (or another early item) and progressing associatively from one letter to the next until one of the two target items is reached.

The alternative view is that the subject can extract ordinal information directly from each letter, so that the order of $H$ and $R$ can often be determined without the aid of associative chaining. A spatial representation of the alphabet (say, from left to right) is one mechanism that could support direct access to ordinal information. Obviously, both methods of obtaining positional information could be available to the subject (see Hamilton \& Sanford, 1978; Lovelace \& Snodgrass, 1971), who might employ one or the other depending on the task demands and the prevailing context.

In any case, to evaluate the plausibility of the associative chain theory as a complete account of the monkey's representational capacity for sequential events, D'Amato and Colombo (1989b) introduced wild-card items in the serial learning task. Monkeys familiar with the ABCDE sequence were trained with a wild-card item (W) that could be substituted for any one of the usual "baseline" items, in effect creating five additional sequences, WBCDE through ABCDW. In a second experiment, two wild cards (X and $Y$ ) were used, generating 10 different sequences (e.g., AXYDE, XBCYE). The argument, in brief, was that because the wild cards did not occupy fixed sequential positions, accounting for reasonably competent wild-card performance on the basis of associative relations alone was highly implausible; attributing some knowledge of ordinal position to the monkeys was a more viable alternative.

The results obtained by D'Amato and Colombo (1989b) did not support the associative chain theory, and thus raised the issue of how to accommodate knowledge of ordinal position representationally. As suggested above, mapping the sequential order of the five-item series onto linear space seems a plausible mechanism (Terrace, 1986). But obtaining direct evidence regarding this hypothesis, which forms the substance of the present report, is not an easy matter. Our approach took advantage of a robust and general phenomenon that emerges when humans judge linearly ordered stimuli-the symbolic distance effect, or more simply, the distance effect.

In a wide variety of situations, human subjects are faster to judge which member of a pair has more (or less) of the attribute along which the test items are ordered the greater the difference (distance) that separates the two items. For example, if subjects are trained that Susan is smarter than Jane, that Jane is smarter than Ann, and so forth for several pairs of individuals, when later tested on all possible pairings, they will judge remote pairs (e.g., Susan/Ann) faster than adjacent pairs (e.g., see Potts, 1974).

More to the present point, the distance effect has been observed with serially organized stimuli or events, for example, letters of the alphabet (Hamilton \& Sanford, 1978; Lovelace \& Snodgrass, 1971) and scripts (Nottenburg \& Shoben, 1980). These results indicate that, at least in humans, items that do not share a common attribute but are associatively or temporally related may also produce the distance-effect phenomenon.

In accommodating the distance effect, many theorists assume that on a test trial the subject extracts positional information directly from each target item (see Holyoak \& Patterson, 1981). In large measure, the distance effect is generated by the fact that the more separated the two items, the easier, and therefore the faster, one can obtain this information and perform the required computation. Associative chaining (serial scanning) is generally regarded as an implausible explanation because, among other things, it incorrectly implies that the distance effect is due solely to the circumstance that the greater the separation between the items of a pair, the faster on average a serial scan will locate the first item (Banks, 1977). Manifestation of the distance effect would therefore appear to provide reasonably strong evidence that subjects have extracted positional information directly from the target items, and would be consistent with the spatial recoding hypothesis.

With regard to the pairwise test administered to our monkeys, evidence for a distance effect would take the form of decreasing first-item response latency across Pairs $\mathrm{BC}, \mathrm{BD}$, and $\mathrm{BE}$, and across Pairs $\mathrm{CD}$ and $\mathrm{CE}$. Pairs that included Item A might not show a distance effect because of the very short response latencies likely to be elicited by that item. (Second-item response latencies are not directly relevant to this issue.) Reexamining first-item latencies of the pairwise test reported by D'Amato and Colombo (1988), we found little sign of a distance effect. However, it seemed possible that the extensive wild-card training received by the monkeys of the D'Amato and Colombo (1989b) study might have sharpened the subjects' knowledge of ordinal position to the point that a second pairwise test would now reveal a distance effect. Experiment 1 addressed this issue.

\section{EXPERIMENT 1}

\section{Method}

\section{Subjects}

Two female New World monkeys (Cebus apella), Fifi and Goldy, who completed the D'Amato and Colombo (1988, 1989b) studies, served as subjects. Fifi was wild-born and 24 years of age; Goldy, laboratory-born, was between 8 and 9 years of age at the start of the study.

The subjects, individually housed, received a single feeding of Purina Monkey Chow (No. 5045) delivered approximately $2-4 \mathrm{~h}$ following an experimental session, adjusted to an amount that sup- 
ported reliable performance. Occasionally supplemented with fresh fruit, the rations were sufficient to maintain the subjects at $91 \%-95 \%$ of their free-feeding body weights.

\section{Apparatus}

The apparatus was a Lehigh Valley monkey operant chamber (Model 1317), the same as previously employed (D'Amato \& Colombo, 1988, 1989b). The front panel housed five inline stimulus projectors (IEE Model 1071) located at the corners and center of a 12-cm square. Each projector was fitted with a transparent plastic key that served as the response mechanism. The chamber was illuminated by an overhead houselight, which could be dimmed by adding a 500- $\Omega$ resistor in series with the bulb. Extraneous sounds were masked by a white noise and an exhaust fan, which together measured $75 \mathrm{~dB}$ (re $0.0002 \mu \mathrm{bar}$ ) on a Simpson sound-level meter (Model 886, C scale) located in the center of the experimental chamber. Data storage and programming of trial events and stimulus presentations were controlled by a PDP 8/e minicomputer and a Commodore PET microcomputer and disk unit.

The stimuli consisted of a red disk $25 \mathrm{~mm}$ in diameter, and five forms: a circle, a vertical line, a plus (superimposed vertical and horizontal lines), an hourglass (superimposed inverted and upright triangles), and a small solid dot. All form stimuli appeared as white figures on a black background and, except for the circle and the dot, were composed of $1.5 \times 17 \mathrm{~mm}$ lines. The circle, also composed of a $1.5-\mathrm{mm}$ line, was $17 \mathrm{~mm}$ in diameter; the dot was $6 \mathrm{~mm}$ in diameter. For Fifi, the stimuli serving as Items A, B, C, D, and $E$ were, respectively, circle, red disk, dot, vertical line, and hourglass; for Goldy, they were circle, plus, dot, red disk, and hourglass. These were the same assignments as employed by D'Amato and Colombo (1988, 1989b).

\section{Procedure}

Retraining on the five-item series. Although a year had elapsed between the end of the wild-card study (D'Amato \& Colombo, 1989b) and the beginning of the present experiment, the subjects required only two 40-trial retraining sessions on the ABCDE series to reach a very high performance level. On the second session, Fifi correctly completed the five-item sequence on $90.0 \%$ of the trials; for Goldy, this figure was $97.5 \%$. During all phases of this and the subsequent experiment, one training or test session was given daily.

The pairwise test. The procedures used by D'Amato and Colombo (1988) in their pairwise test were followed rather closely. Each test session consisted of 40 trials, four with each of the 10 test pairs. A typical test trial proceeded as follows. At the end of a 15-sec intertrial interval (ITI), the houselight was illuminated and, $2 \mathrm{sec}$ later, the two stimulus items appeared simultaneously on any two of the five projectors. The first correct response (pressing the response key on which the first item of the pair appeared) sounded a $1 / 6$-sec feedback tone. Repeated presses were not considered errors, but only the first press resulted in the feedback tone. Following a response to the second item of the pair, a 190-mg Noyes banana pellet was delivered, the stimulus display was extinguished, and the ITI (signaled by dimming of the houselight and illumination of a dipper-well light) was begun. An error-pressing the second item of the pair before responding to the first-began a 60 -sec timeout period, indicated by the dimmed houselight alone. Response latency was measured in units of $20 \mathrm{msec}$.

The experimental design was based on one test session, followed by two baseline sessions on the five-item series, and finally a second test session. Because Fifi made numerous errors on her initial test session, the data were discarded and the session was repeated. Thus, as in the earlier pairwise test, each of the 10 test pairs was presented a total of eight times.

\section{Results and Discussion}

Very few errors were committed during the pairwise test, Fifi and Goldy scoring $92.5 \%$ and $93.8 \%$ correct responses, respectively. Following D'Amato and Colombo (1988), for each test session a median first-item response latency was calculated for each test pair, on the basis of correct trials only. The two median latencies generated for each of the 10 test pairs were then averaged to obtain a single value for each pair. Using a similar procedure, average second-item response latencies were also calculated for each test pair.

The first question addressed was whether the latency relationships obtained in the pairwise test conducted 2 years earlier (D'Amato \& Colombo, 1988) still held. Figure 1 shows that the relationship between first-item response latency and serial position of the first item was still strongly in force (see D'Amato \& Colombo, 1988, Figure 5). A randomized blocks analysis of variance (ANOVA) revealed first-item position to be significant $[F(3,3)=15.57, p<.03]$, with the linear-trend component accounting for $89 \%$ of the variation.

As we found earlier, second-item response latency increased in an orderly way with increases in the number of missing items separating the items of a test pair, though the strength of the relation was much attenuated. The number of missing items did not prove statistically reliable, but, since $98 \%$ of the variation was in the linear component, this contrast was significant $[F(1,3)=19.42$, $p<.03]$. Because a correct second-item response was unavoidable following a correct first-item response, it is likely that on some trials the monkeys fell into the default strategy of pressing the second item without attempting to match it to a represented item.

The strong dependence of first-item response latency on the serial position of the first item raises an interpretive problem with regard to the distance effect. Compar-

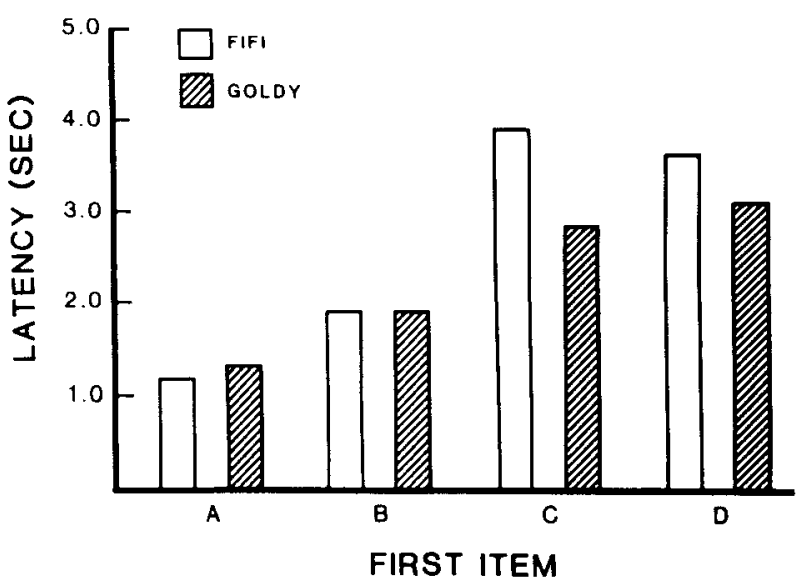

Figure 1. Mean first-item response latency for Fifi and Goldy to test pairs whose first item was A, B, C, or D in Experiment 1. 
ison of such latencies for test pairs in which the number of missing items is 0 (Pairs $\mathrm{AB}, \mathrm{BC}, \mathrm{CD}$, and $\mathrm{DE}$ ), 1 (Pairs AC, BD, and CE), 2 (Pairs AD and BE), or 3 (Pair AE) would appear to provide a straightforward way of detecting the presence of a distance effect. Unfortunately, though, this comparison confounds item separation with first-item location.

To illustrate this point, Goldy's mean first-item response latencies for separations of $0,1,2$, or 3 items, calculated from the actual value of each of the test pairs listed above, were $2.34,1.98,1.56$, and $1.33 \mathrm{sec}$, respectivelyapparently, strong evidence of a distance effect. However, when the value assigned to each test pair was the mean first-item latency based on all pairs having the same first item (see Figure 1), the corresponding quantities were $2.29,2.02,1.61$, and $1.31 \mathrm{sec}$. Obviously, sequential position of the first item of a test pair accounts for virtually all of the variation in the first set of numbers. As implied earlier, such a result is consistent with the serial scan (associative chain) account of the distance effect.

The first-item response latencies presented in Figure 2 (right panel) are free of the previous problem because comparisons are across test pairs that have the same first item. As is apparent, the depicted data provide little evidence of distance effect in either subject. Although Fifi's response latency to Item $B$ decreased across Test Pairs $B C, B D$, and $B E$, her latency to Item $C$ was much higher for Test Pair CE than for Pair CD. Goldy's response latency to Item $\mathrm{C}$ was shorter for Pair CE than for Pair CD, but she did not show the expected pattern with regard to Item B. Moreover, in human studies, the distance effect is often accompanied by a nonmonotonic or even an inverted- $U$ function relating response latency to successive adjacent test pairs (Banks, 1977). The left panel of Figure 2 shows that the first-item response latencies associated with adjacent test pairs were not of this general form.

The rather striking replication of the function relating first-item response latency to first-item position (Figure 1) provides a clue as to why the distance effect has proved elusive in our situation. Let us suppose that the monkeys have available to them both an associative chain and a spatial array representation of the ABCDE series. If such were the case, intensive past experience with the five-item series, requiring as it does that the monkeys start at Item A and progress item by item through the series, should bias them toward accessing the associative chain representation. When suddenly confronted with pairs of test items, they naturally would tend to continue accessing the associative chain representation, which would greatly reduce

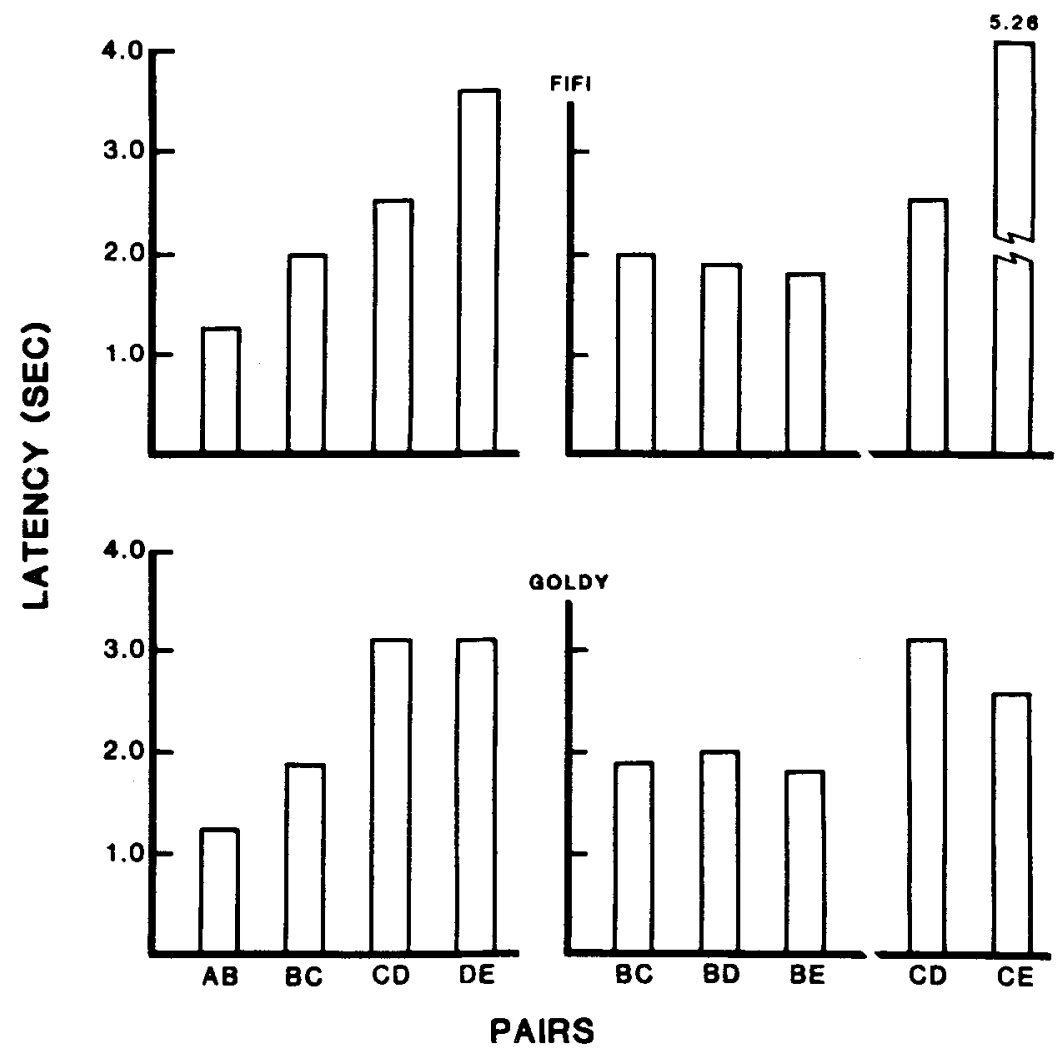

Figure 2. Right panel: Fifi and Goldy's first-item response latencies for Test Pairs BC, BD, and BE, and for Pairs CD and CE. A distance effect would be revealed by decreasing response latencies across both sets of test pairs. Left panel: First-item response latencies for the four adjacent test pairs AB, BC, CD, and DE. Normally, the distance effect is accompanied by a nonmonotonic or an inverted-U latency function across successive adjacent pairs. 
the chances of observing a distance effect. Even in the wild-card studies (D'Amato \& Colombo, 1989b), a substantial proportion of trials were baseline $\mathrm{ABCDE}$ trials; moreover, on the wild-card trials themselves, the monkeys could have relied mainly on the associative chain representation, resorting to ordinal information only to correctly position the wild cards.

The procedure employed in many related studies of the distance effect is to train the subjects on adjacent pairs and then assess their performance on these and remote pairs, a procedure that does not bias the subject in the direction of associative chaining. There is, indeed, some evidence that squirrel monkeys display a distance effect when trained only on adjacent pairs prior to testing (McGonigle \& Chalmers, 1986).

\section{EXPERIMENT 2}

In the present experiment, we attempted to encourage Fifi and Goldy to access their respective spatial representations of the five-item series, if indeed they possessed one, by training them on Adjacent Pairs $\mathrm{AB}, \mathrm{BC}, \mathrm{CD}$, and $\mathrm{DE}$ prior to testing with all 10 pairs. Two other changes were instituted. To increase experimental precision, stimulus pairs were presented only on the upper two projectors, and to move the procedure closer to other studies of the distance effect, second-item responses were no longer required. We thought the contextual change introduced by the latter modification might also help the monkeys relinquish the associative chain processing mode. Finally, a 3rd subject was added to the study.

\section{Method}

\section{Subjects and Apparatus}

In addition to Fifi and Goldy, Jane, a laboratory-born female $\mathrm{Ce}$ bus apella monkey between 8 and 9 years of age, served as a subject. Jane had completed the D'Amato and Colombo (1988) experiment and had begun the wild-card study (D'Amato \& Colombo, $1989 \mathrm{~b}$ ) but, because she performed poorly on wild-card sequences, her training was terminated at an early stage. The stimuli that served as Items A, B, C, D, and E were the same for Jane as those assigned to Goldy-circle, plus, dot, red disk, and hourglass, respectively. Jane had been away from the serial learning task for 14 months. In order to have her enter the experiment on a reasonably comparable footing with the other subjects, she was given 11 retraining sessions on the five-item series; she scored $70 \%$ correct trials over the last two of these sessions.

The apparatus was the same as in Experiment 1.

\section{Procedure}

Training on adjacent pairs consisted of 40-trial sessions, 10 trials with each of the $\mathrm{AB}, \mathrm{BC}, \mathrm{CD}$, and $\mathrm{DE}$ pairs, quasirandomly intermixed. The tasks posed by the four stimulus pairs may be viewed as conditional discriminations in which the status of Items $\mathbf{B}, \mathbf{C}$, and $\mathrm{D}$ is governed by the identity of the paired item (e.g., $\mathrm{A}+\mathrm{B}-$ vs. $\mathrm{B}+/ \mathrm{C}-$ ). Testing differed from training only in that all 10 pairs were presented, each pair being represented four times in the 40trial test sessions. Of course, for the six (remote) pairs not presented during training, responding to the item with the earlier sequential position in the ABCDE series was the correct response.

As already noted, the stimuli appeared on the top two of the five projectors, with the left/right orientation of the items of a pair balanced within each training and test session. The sequence of events of a trial was modified as follows. At the end of a $15-\mathrm{sec}$ ITI, the houselight was illuminated, and following completion of four responses on a microswitch located on the front wall of the chamber, a pair of items was presented on the upper projectors. A single response to the correct item was reinforced with the usual 190-mg Noyes banana pellet; pressing the incorrect item resulted in the customary 60 -sec timeout.

A training/test cycle consisted of a series of training sessions al ways followed by 10 test sessions. Goldy, who very early in training displayed a strong nonmonotonic latency function across adjacent pairs, received one such cycle, on the basis of only two training sessions. Jane was given two cycles, with nine training sessions in the first cycle and five in the second. Fifi received the same treatment as Jane, except that two of the training sessions in the first cycle were interposed between Sessions 5 and 6 of the test sessions. The reason for increasing the number of test sessions from the two used in the pairwise test was to generate sufficient data to permit individual-subject analyses.

\section{Results and Discussion}

The 10 test sessions of a cycle were arranged in blocks of two sessions, and a median latency was calculated within each block for each of the 10 test pairs, on the basis of correct trials only. Averaging the five available medians produced a single mean latency for each test pair. In the terminology of the pairwise test of Experiment 1, these correspond to first-item response latencies.

Neither Fifi nor Jane displayed a distance effect in their first cycle of test sessions, although both performed at a high level of accuracy ( $95 \%$ correct) and Jane produced a nonmonotonic latency function across the adjacent test pairs on her later training sessions. Response latency to Item $B$ did not decrease with increasing separation of the paired items, as both subjects responded faster to Test Pair BC than to Pair BD. In addition, Fifi responded faster to Pair $C D$ than to Pair $C E$. It seemed possible that the nine training and 10 test sessions of their first cycle might not have been sufficient to completely divert these subjects from the associative chain mode of processing, which was the rationale for the second training/test cycle.

Figure 3 presents the results of Fifi and Jane from their second cycle of test sessions, as well as those of Goldy, who received only one training/test cycle. All 3 subjects showed at least qualitative evidence of a distance effect (Figure 3, right panel). Without exception, response latency decreased across Test Pairs BC, BD, and BE, and across Pairs $C D$ and $C E$. Thus, holding serial location of the first item fixed, response latency decreased as the separation between the two items of a test pair increased. The differences were very small in the case of Fifi, but their pattern represents a marked change over what was observed in her first cycle. As expected, response latencies to pairs that included Item A were uniformly low, with virtually no overlap with non-A pairs, and they were unrelated to item separation.

For each subject, a randomized blocks ANOVA was applied to the latencies produced by Test Pairs BC, BD, and $\mathrm{BE}$, with blocks (5) and separation (3) as factors. Separation was a statistically significant factor in the case 

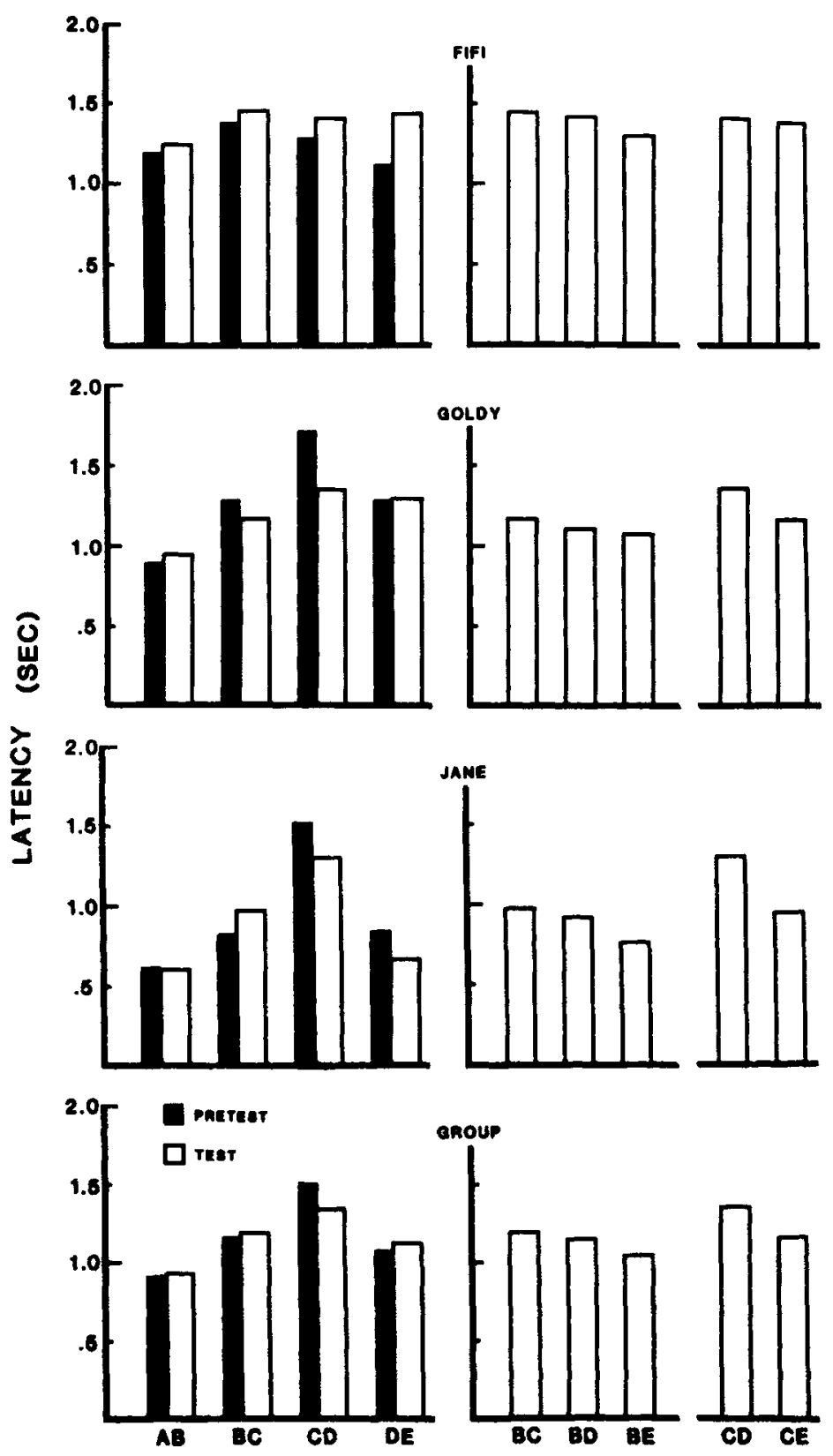

PAIRS

Figure 3. Individual-subject and group response latencies obtained with the modified training/testing procedure of Experiment 2. Results from the five critical test pairs are presented in the right panel. Response latency to the four successive adjacent pairs are shown in the left panel, both for test sessions and for the last training session prior to testing (pretest). The results of Fif and Jane are based on the 10 test sessions of their second training/test cycle; Goldy received only one such cycle.

of Goldy and Jane $[F(2,8)=8.16$ and 8.99 , respectively, $p<.02$ ], but not for Fifi. The group data were analyzed by a similar ANOVA, with subjects (3) and separation (3) as factors, and separation was again statistically significant $[F(2,4)=12.19, p<.02]$.

The individual-subject latency differences across Test Pairs CD and CE were evaluated by paired $t$ tests, which showed that the difference was significant in the cases of Goldy and Jane $[t(4)=3.14, p<.04$, and $t(4)=6.24$, $p<.005$, respectively], but not for Fifi. In part because Fifi's average difference score was much smaller than those of the other 2 subjects, the group difference between Pairs CD and CE, also evaluated by a paired $t$ test, was not statistically reliable $[t(2)=1.88, p=.20]$.

The left panel of Figure 3 presents response latencies on adjacent pairs obtained during the last training session (pretest) and during the subsequent 10 test sessions (test). For all 3 subjects, a pattern of nonmonotonic latencies 
across adjacent pairs was observed in the training session, strongly so in the cases of Goldy and Jane. Nonmonotonicity was absent from Fifi's test data, and though greatly reduced in the case of Goldy, a test of quadratic trend was statistically reliable $[F(1,12)=9.92, p<.01]$; Jane's adjacent-pair latencies were again strongly nonmonotonic.

The present results provide the first consistent evidence for a distance effect in our monkeys. Latencies uniformly decreased across Test Pairs BC, BD, and BE, and across Pairs $C D$ and $C E$. With one exception, nonmonotonicity across adjacent pairs was observed during both training and testing. And, interestingly, the subject who displayed the weakest nonmonotonicity, Fifi, also produced the least evidence of a distance effect.

Nevertheless, the fact that pairs that included Item E were involved in the critical comparisons might be cause for some concern, as one might expect uniformly short latencies to such pairs. As Figure 3 shows, this was not the case. Both Goldy and Jane, for example, responded a bit faster to Pair BD than to Pair CE. Moreover, reasonable support for the distance effect can be found in the interior pairs, BC and BD. A paired $t$ test assessed the latency difference produced by these pairs across all 3 subjects, and though the difference was small $(50 \mathrm{msec})$ and the degrees of freedom few, it proved reliable $[t(2)$ $=5.54, p<.05$ ]. A similar test applied to Goldy's data was significant $[t(4)=4.35, p<.02]$, all five difference scores favoring the BD pair. The difference score of Jane's first test block was atypical in that it was by far the largest of the five and the only one that showed a shorter latency for Pair BC. Without it, the BC-BD comparison was highly significant $[t(3)=9.25$, $p<.003$ ]. Fifi's BC-BD difference was not reliable.

Finally, although response latency for Fifi and Goldy was directly related to the serial position of the first item, the association was greatly attenuated relative to that observed in the pairwise test of Experiment 1, and was not statistically reliable across the 2 subjects $[F(3,3)=5.84$, $p>.05]$. Whereas in Experiment 1, Fifi's first-item response latency to Item $D$ was $2.45 \mathrm{sec}(308 \%)$ longer than that to Item A (Figure 1), it was only $0.16 \mathrm{sec}(13 \%)$ longer in the present experiment; for Goldy the corresponding values were $1.79 \mathrm{sec}(237 \%)$ and $0.36 \mathrm{sec}$ (39\%). In the case of Jane, the function relating response latency to first-item location was sharply nonmonotonic. These results are consistent with the assumption that the present procedure served to reduce the subjects' reliance on the associative chain processing mode.

\section{GENERAL DISCUSSION}

The results of Experiment 2, in conjunction with those of the wild-card study (D'Amato \& Colombo, 1989b), provide substantial evidence that monkeys can extract positional information directly from serially related items. Our interpretation of these findings is that monkeys have at their disposal both an associative chain and a spatial representation of the $\mathrm{ABCDE}$ series. They access one or the other for processing in accordance with past experience and current task demands. The rapidity with which the monkeys appear to have shifted from the associative chain to the spatial representation in Experiment 2 , despite intensive previous experience running off the $\mathrm{ABCDE}$ series, suggests that the latter representation is readily available to them for processing.

As for the nature of the associative chain and spatial representations, whether they constitute separate representations or merely different manifestations of the same trace system, Lashley's (1951) observations of some four decades ago are relevant. Discussing the interaction of temporal and spatial systems, Lashley wrote: "When the memory trace is formed it is integrated with directional characters of the space system, which give it position in reference to other associated traces," adding, "These space characters of the memory trace can be scanned by some other level of the coordinating system and so transformed into succession" (p. 129).

Applied to our situation, Lashley's (1951) analysis suggests that the representation (trace, in his terms) of each item of the temporally organized $\mathrm{ABCDE}$ series incorporates a spatial marker, and that these markers can be interpreted by a higher level control system that can then activate them in sequence or, if need be, even in reverse sequence (in the case of humans, at least). Within this formulation, processing based on the associative chain or on the spatial representation could be viewed as simply two different ways of querying the spatial markers. In the first, the control system accesses the spatial markers to sequence the represented items of the $\mathrm{ABCDE}$ series, thus allowing the represented items to be correctly matched to the displayed items. In the second, one or more displayed items are first registered and their associated spatial markers are then read out, providing the monkey with direct information regarding position.

A major problem with this interpretation is that the information provided by spatial markers about serial position is, as we have seen, not very exact, which raises the important question as to how relatively imprecise positional cues could by themselves generate precise sequencing of a series' items. It seems more plausible to assume that the "glue" for precise sequencing comes from associative linking of the represented items; however, since the latter are are also coded as a spatial array, each item acquires a spatial marker. By this account, when monkeys access the associative-chain mode to sequence the displayed items, they make little use of the positional cues provided by spatial markers, relying primarily on the associative linkages. However, when one or more items are inputted without first accessing the associative chain, the attached spatial markers come into play, providing the monkeys with direct, if relatively imprecise, information regarding ordinal position.

The ability of animals to represent a temporal sequence of events spatially, and thus gain direct access to positional information, can greatly extend their cognitive reach. The distance effect is one such illustration, but there probably are many others. For example, Capaldi and 
Miller (1988) recently proposed that rats trained on a series of reinforced and nonreinforced events in a runway are capable of counting successive reinforcing events, and of doing so in an abstract way. That is, they can enumerate qualitatively different reinforcers. Although it is clear from Capaldi and Miller's results that rats are very good at tracking reinforcing events, we think it is possible that much of the evidence presented in support of counting can be interpreted in terms of the rat's capacity to form spatial representations of serial events. The positional information available in such representations could, in conjunction with other factors, account for many aspects of the rats' transfer performances, which, on early transfer trials, often seem to lack sufficient precision to demand a true counting ability.

Another illustration comes from the interesting work of Hulse and his associates on rule learning by rats (e.g., Fountain \& Hulse, 1981; Hulse \& Dorsky, 1979). Rats trained on a sequence of trials in which the reward magniiude (number of food pellets) assigned to each trial decreased monotonically (e.g., 14-7-3-1-0) learned to anticipate the final, 0 , value faster than did rats exposed to exactly the same reward values but not in a monotonic order (e.g., 14-1-3-7-0). These and related findings have been taken as evidence that rats are capable of learning rules of the form $E(i+1)<E(i)$, where the two terms refer to the reward magnitudes on successive trials of the sequence.

It appears to us that ascribing such rule learning to rats attributes both too much and too little to the rat's cognitive competence: too much, because the capacity for abstraction implied by that attribution seems to exceed even the monkey's ability to perceive abstract relations (D'Amato, 1988; D'Amato \& Colombo, 1989a; D'Amato, Salmon, \& Colombo, 1985); too little, because, as Roitblat, Pologe, and Scopatz (1983) have clearly shown in an experiment resembling our wild-card studies, rats learn something about the ordinal position of the different reward magnitudes of a sequence. Acquisition of such ordinal information does not seem to be directly implied by the putative rule learning.

We suspect that rather than learning about the pattern of the reward magnitudes, rats learn about their sequence. As in the case of our monkeys, rats very likely form both an associative chain and a spatial representation of the relevant sequence. But because the items (reward magnitude) of the sequence differ only quantitatively, the positional information available in the spatial representation will be less precise than is the case for the $A B C D E$ series (at least until the sequence is well learned) and therefore subject to generalization-induced errors. We believe that many of the transfer results of Hulse and his colleagues, including the basic result described above, can be accommodated by this approach.

Finally, that a wide array of cognitive competencies in animals, ranging from cognitive maps to the distance effect, might be mediated by a similar mechanismtranslation of associative chain representations of temporal sequences into spatial arrays-is an interesting possibil- ity worthy of further exploration, from both the behavioral and the neuroscience directions.

\section{REFERENCES}

BANks, W. P. (1977). Encoding and processing of symbolic information in comparative judgments. In G. H. Bower (Ed.), The psychology of learning and motivation: Advances in theory and research (Vol. 11, pp. 101-159). New York: Academic Press.

Capaldi, E. J., \& Miller, D. J. (1988). Counting in rats: Its functional significance and the independent cognitive processes that constitute it. Journal of Experimental Psychology: Animal Behavior Processes, 14, 3-17.

D'AмAто, M. R. (1988). A search for tonal pattern perception in cebus monkeys: Why monkeys can't hum a tune. Music Perception, $5,453-480$.

D'Aмato, M. R., \& Colombo, M. (1988). Representation of serial order in monkeys (Cebus apella). Journal of Experimental Psychology: Animal Behavior Processes, 14, 131-139.

D'Amato, M. R., Colombo, M. (1989a). On the limits of the matching concept in monkeys (Cebus apella). Joumal of the Experimental Analysis of Behavior, 52, 225-236.

D'Aмлто, M. R., \& Colombo, M. (1989b). Serial learning with wild card items by monkeys (Cebus apella): Implications for knowledge of ordinal position. Journal of Comparative Psychology, 103, 252-261.

D'Amato, M. R., SAlmon, D. P., \& Colombo, M. (1985). Extent and limits of the matching concept in monkeys (Cebus apella). Journal of Experimental Psychology: Animal Behavior Processes, 11, 35-51.

FounTAIN, S. B., \& HULSE, S. H. (1981). Extrapolation of serial stimulus patterns by rats. Animal Learning \& Behavior, 9, 381-384.

Hamiton, J. M. E., \& Sanford, A. J. (1978). The symbolic distance effect for alphabetic order judgements: A subjective report and reaction time analysis. Quarterly Journal of Experimental Psychology, 30, 33-43.

HolyonK, K. J., \& Patterson, K. K. (1981). A positional discriminability model of linear-order judgments. Journal of Experimental Psychology: Human Perception \& Performance, 7, 1283-1302.

Hulse, S. H., \& DorSky, N. P. (1979). Serial pattern learning by rats: Transfer of a formally defined stimulus relationship and the significance of nonreinforcement. Animal Learning \& Behavior, 7, 211-220.

LASHLEY, K. S. (1951). The problem of serial order in behavior. In L. A. Jeffress (Ed.), Cerebral mechanisms in behavior (pp. 112-136). New York: Wiley.

Lovelace, E. A., SNodgrass, R. D. (1971). Decision times for alphabetic order of letter pairs. Journal of Experimental Psychology, 88, 258-264.

McGonigle, B., \& Chalmers, M. (1986). Representations and strategies during inference. In T. Myers, K. Brown, \& B. McGonigle (Eds.), Reasoning and discourse processes (pp. 141-164). London: Academic Press.

NotTenburG, G., \& SHoben, E. J. (1980). Scripts as linear orders. Journal of Experimental Social Psychology, 16, 329-347.

PotTs, G. R. (1974). Storing and retrieving information about ordered relationships. Journal of Experimental Psychology, 103, 431-439.

RoItBlat, H. L. (1982). The meaning of representation in animal memory. Behavioral \& Brain Sciences, 5, 353-406.

Roitblat, H. L., Pologe, B., \& SCopatZ, R. A. (1983). The representation of items in serial position. Animal Learning \& Behavior, 11, 489-498.

SlameckA, N. J. (1985). Ebbinghaus: Some associations. Journal of Experimental Psychology: Leaming, Memory, \& Cognition, 11, 414-435.

Straub, R. O., * Terrace, H. S. (1981). Generalization of serial learning in the pigeon. Animal Learning \& Behavior, 9, 454-468.

TerRace, H. S. (1986). A nonverbal organism's knowledge of ordinal position in a serial learning task. Journal of Experimental Psychology: Animal Behavior Processes, 12, 203-214.

TeRraCe, H.S. (1987). Chunking by a pigeon in a serial learning task. Nature, 325, 149-151.

(Manuscript received March 29, 1989; accepted for publication May 24, 1989.) 